\title{
Methylation of the Sclerostin Gene in Serum Free DNA: A New Bone Biomarker?
}

\author{
Alvaro Del Real, ${ }^{1}$ Flor M. Perez-Campo, ${ }^{2}$ M. Isabel Perez-Nuñez, ${ }^{3}$ Carolina Sañudo, ${ }^{1}$ Ana Santurtun, ${ }^{4}$ \\ Carmen Garcia-Ibarbia, ${ }^{1}$ M. Teresa Garcia-Unzueta, ${ }^{5}$ Mario F . Fraga, ${ }^{6}$ Agustin F . Fernandez, ${ }^{7}$ \\ M. Carmen Valero, ${ }^{1}$ Esther Laguna, ${ }^{2}$ and José A. Riancho ${ }^{1}$
}

Introduction: Cell-free DNA (cfDNA) methylation is an important molecular biomarker, which provides information about the regulation of gene expression in the tissue of origin. There is an inverse correlation between SOST methylation and expression levels.

Methods: Thus, we analyzed SOST promoter methylation in serum cfDNA and compared it with DNA from blood and bone cells from patients undergoing hip replacement surgery. We also measured cfDNA methylation in 28 osteoporotic patients at baseline and after 6 months of antiosteoporotic therapy (alendronate, teriparatide, or denosumab).

Results: SOST methylation levels in serum cfDNA were very similar to those of bone-derived DNA $(79 \%-12 \%$ and $82 \%-7 \%$, respectively), but lower than methylation levels in blood cell DNA $(87 \%-10 \%)$. Furthermore, there was a positive correlation between individual values of DNA methylation in serum and DNA methylation in bone. No differences in either serum sclerostin levels or SOST methylation were found after 6-month therapy with antiosteoporotic drugs.

Conclusions: Our results suggest that serum cfDNA is not originated from blood cells, but from bone. However, since we did not confirm changes in this marker after therapy with bone-active drugs, further studies showing a correlation between bone changes of SOST expression in bone and SOST methylation in cfDNA are AU4 c needed to confirm its potential role as a bone biomarker.

Keywords: biomarker, DNA methylation, cell free DNA, osteoporosis, bone

Introduction

$\mathrm{B}$ iomarker research aims at finding substances that can be measured in blood or other body fluids and would, in some way, reflect the state of relevant organs, so that they can be used as a noninvasive procedure to assess disease risk, disease presence, or disease evolution, with or without therapy. This is particularly useful for disorders of inner organs, such as the skeleton, which cannot be easily sampled without invasive interventions. A variety of proteins have been traditionally used as biomarkers. More recently, the discovery of circulating nucleic acids has expanded the biomarker landscape (Connolly et al., 2016). In this regard, cell-free
DNA (cfDNA) has been identified in serum and is currently used, in pregnant women, to detect fetal chromosomal abnormalities (Biró et al., 2018; Goldwaser and Klugman, 2018). cfDNA is also being actively investigated in neoplastic diseases, as a mean to detect and follow up a variety of cancers, and so the "liquid biopsy" is being rapidly introduced in oncological clinical practice (Yamashita et al., 2018). The utility of cfDNA in oncology is based upon two facts. First, the amount of cfDNA is usually markedly increased in the presence of tumors, thus facilitating the analysis. Second, tumors tend to accumulate mutations and epimutations that allow to establish their signature in circulating cfDNA. However, there are currently insufficient data

\footnotetext{
${ }^{1}$ Department of Internal Medicine, Hospital Universitario Marqués de Valdecilla-IDIVAL, University of Cantabria, Santander, Spain

${ }^{2}$ Department of Molecular Biology, University of Cantabria-IDIVAL, Santander, Spain.

${ }^{3}$ Department of Traumatology/Hospital U M Valdecilla, University of Cantabria, Santander, Spain.

${ }^{4}$ Unit of Legal Medicine, Department of Physiology and Pharmacology, University of Cantabria, Santander, Spain.

${ }^{5}$ Service of Clinical Biochemistry, Hospital U.M. Valdecilla, University of Cantabria, IDIVAL, Santander, Spain.

${ }^{6}$ Cancer Epigenetics Laboratory, Institute of Oncology of Asturias (IUOPA), Nanomaterials and Nanotechnology Research Center (CINN-CSIC), University of Oviedo, Asturias, Spain.

${ }^{7}$ Institute of Oncology of Asturias (IUOPA), ISPA-HUCA. Fundación para la Investigación Biosanitaria de Asturias (FINBA), Asturias, Spain.
} 
about the potential use of cfDNA in non-neoplastic disorders, with the exception of a few studies exploring its role in monitoring transplant rejection (Zou et al., 2017; LehmannWerman et al., 2018).

Sclerostin, encoded by the SOST gene, is a major regulator of bone remodeling. Although low-level SOST expression is detected in several tissues, sclerostin is considered a bonespecific protein (Weivoda et al., 2017). In the bone microenvironment, sclerostin synthesis is apparently limited to the osteocytes (Delgado-Calle et al., 2017). SOST is a relatively small gene, with only two exons. Interestingly, there is an enhancer region located about $50 \mathrm{~kb}$ apart from the promoter and coding sequence, which mediates the effect of some transcription factors, such as MEF2C. In a previous study, we demonstrated an inverse correlation between SOST methylation and gene expression (Delgado-Calle et al., 2012). Also, DNA methylation at the SOST promoter is related to gene expression in human bone samples (Reppe et al., 2015). Therefore, although the regulation of SOST transcription is not completely understood, there is evidence that the methylation status of the SOST promoter plays a critical role in the activation/repression of SOST transcription. Hence, we undertook this study to explore whether SOST methylation marks in cfDNA revealed a bone or a blood signature. Should the former be the case, then it could represent a convenient mean of exploring sclerostin synthesis in the skeleton.

Methods

\section{Patient selection}

Two groups of patients were studied. The first one included 33 patients undergoing hip replacement surgery due to osteoporotic hip fractures or hip osteoarthritis. From each patient, bone tissue, blood, and serum samples were obtained

T1 c for analysis (Table 1). The second group included 28 osteoporotic women (age $=74-8$ years) who were to begin therapy. Serum samples were obtained at baseline and 6 months after the start of therapy with alendronate $(n=10 ;$ age $=73$ $-7)$, denosumab $(n=10$; age $=71-8)$, or teriparatide $(n=8$; age $=78-8)$, as prescribed by their physician, as clinically

$\mathrm{T} 2 \mathrm{c}$ indicated (Table 2). The study was approved by the IRB (Comité de Etica en Investigación Clínica de Cantabria; Acta 25/2012) and patients provided written informed consent.

\section{DNA isolation}

Trabecular bone samples from the central part of femoral heads were obtained with a trephine. They were snap-frozen in liquid nitrogen and homogenized with a polytron homogenizer in lysis buffer and proteinase K. After an overnight incubation at $55^{\circ} \mathrm{C}$, DNA was extracted with phenol: chloroform:isoamyl alcohol, as previously described (DelgadoCalle et al., 2012).

Serum cfDNA was isolated from two $1 \mathrm{~mL}$ aliquots of serum, processed in parallel. So for each patient, $2 \mathrm{~mL}$ of serum was used for the analysis. To each aliquot, we added $500 \mathrm{~mL}$ of lysis buffer (Tris-HCl, EDTA, sodium acetate, and SDS $)$ and $5 \mathrm{~mL}$ of proteinase $\mathrm{K}(20 \mathrm{mg} / \mathrm{mL})$ in a $15 \mathrm{~mL}$ falcon tube. This mix was incubated for $1 \mathrm{~h}$ in a water bath at $56^{\circ} \mathrm{C}$. DNA isolation was performed with phenol:chloroform: isoamyl alcohol (Fisher ${ }^{\circledR}$ ), using PLG falcon tube (Qiagen ${ }^{\circledR}$ ), by centrifuging at $4500 \mathrm{rpm}$ for $30 \mathrm{~min}$. DNA was finally
Table 1. Patients Used for Baseline Analysis of SOST Methylation

\begin{tabular}{lllllll}
\hline Patient & Disease & Sex & Age & Serum & Bone & Blood \\
\hline 1 & OA & F & 66 & Yes & Yes & Yes \\
2 & OA & F & 70 & Yes & Yes & Yes \\
3 & FRX & F & 89 & Yes & Yes & Yes \\
4 & FRX & F & 84 & Yes & Yes & Yes \\
5 & FRX & F & 81 & Yes & Yes & Yes \\
6 & FRX & F & 91 & Yes & Yes & Yes \\
7 & FRX & F & 66 & Yes & Yes & Yes \\
8 & OA & F & 68 & Yes & Yes & Yes \\
9 & FRX & F & 91 & Yes & Yes & Yes \\
10 & FRX & F & 91 & Yes & Yes & Yes \\
11 & OA & M & 67 & Yes & - & - \\
12 & OA & M & 71 & Yes & - & - \\
13 & OA & F & 75 & Yes & - & - \\
14 & OA & F & 73 & Yes & - & - \\
15 & OA & F & 83 & Yes & - & - \\
16 & OA & F & 65 & Yes & - & - \\
17 & OA & M & 65 & Yes & - & - \\
18 & FRX & F & 80 & Yes & - & - \\
19 & OA & F & 84 & Yes & - & - \\
20 & OA & M & 83 & Yes & - & - \\
21 & FRX & M & 82 & Yes & - & - \\
22 & OA & F & 67 & Yes & - & - \\
23 & FRX & F & 86 & Yes & - & - \\
24 & OA & F & 64 & Yes & - & - \\
25 & FRX & F & 83 & Yes & - & - \\
26 & FRX & F & 78 & Yes & - & - \\
27 & OA & F & 73 & Yes & - & - \\
28 & OA & M & 68 & Yes & - & - \\
29 & OA & M & 68 & Yes & - & - \\
30 & OA & M & 62 & Yes & - & - \\
31 & FRX & F & 83 & Yes & - & - \\
32 & FRX & F & 90 & Yes & - & - \\
33 & FRX & M & 90 & Yes & - & - \\
\hline & FR & & & & &
\end{tabular}

F, female; FRX, fractures; M, male; OA, osteoarthritis.

precipitated with 1 volume of isopropanol (*1 mL) and 0.1 volumes of $3 \mathrm{M}$ sodium acetate. Samples were then centrifuged for $1 \mathrm{~h}$, at $13,000 \mathrm{rpm}$ and $4^{\circ} \mathrm{C}$. The pellet (not visible) was cleaned with $70 \%$ of ethanol, followed by centrifugation for $30 \mathrm{~min}$, at $13,000 \mathrm{rpm}$ and $4^{\circ} \mathrm{C}$. Pellet was air dried at room temperature and resuspended with $20 \mathrm{~mL}$ of distilled water.

DNA was also extracted from $1 \mathrm{~mL}$ aliquots of whole blood (drawn into EDTA-containing standard tubes) with the same procedure.

\section{DNA methylation analysis}

The whole DNA volume $(20 \mathrm{~mL})$ was subjected to bisulfite modification with EZ DNA Methylation-Gold Kit (ZymoResearch ${ }^{\circledR}$ ), following manufacturer's instructions. The methylation level of selected CpGs, at the SOST promoter region, was analyzed by pyrosequencing (PyromarkQ24 Advanced System ${ }^{\circledR}$ ). Primers used for PCR amplification and sequencing were designed with the PyroMark assay designer (Primer Forward: 5 -TGGTGGGGTGATAA ATGAATT-3 ; Primer Reverse: 5 ATGAATT-3c; and Primer Sequencing: 5c-ATTTGGTTTG

AGAAATGG-3\$). This procedure was used to examine the methylation of $3 \mathrm{CpGs}$, situated about 300 base pairs from the 
Table 2. Patients Studied at Baseline and After Treatment

\begin{tabular}{|c|c|c|c|c|c|c|c|}
\hline Patient & Age & $\operatorname{Sex}$ & Disease & Treatment & $\begin{array}{l}\text { Fragility } \\
\text { fractures }\end{array}$ & $\begin{array}{l}\text { Lumbar } \\
\text { spine } \\
\text { T-score }\end{array}$ & $\begin{array}{c}\text { Femoral } \\
\text { neck } \\
\text { T-score }\end{array}$ \\
\hline A1 & 83 & $\mathrm{~F}$ & Osteoporosis & Alendronate & Yes & -0.9 & -2.9 \\
\hline A2 & 65 & $\mathrm{~F}$ & Osteoporosis & Alendronate & No & -3.0 & -3.4 \\
\hline A3 & 70 & $\mathrm{~F}$ & Osteoporosis & Alendronate & Yes & -3.3 & -2.3 \\
\hline A4 & 79 & $\mathrm{~F}$ & Osteoporosis & Alendronate & Yes & -2.8 & -2.9 \\
\hline A5 & 72 & $\mathrm{~F}$ & Osteoporosis & Alendronate & Yes & -3.5 & - \\
\hline A6 & 78 & $\mathrm{~F}$ & Osteoporosis & Alendronate & Yes & -3.3 & -1.6 \\
\hline A7 & 72 & $\mathrm{~F}$ & Osteoporosis & Alendronate & No & -3.1 & - \\
\hline A8 & 82 & $\mathrm{~F}$ & Osteoporosis & Alendronate & Yes & -3.7 & -2.1 \\
\hline A9 & 65 & $\mathrm{~F}$ & Osteoporosis & Alendronate & No & - & -2.8 \\
\hline A10 & 67 & $\mathrm{~F}$ & Osteoporosis & Alendronate & No & -3.3 & -3.7 \\
\hline D1 & 58 & $\mathrm{~F}$ & Osteoporosis & Denosumab & No & -2.6 & -1.7 \\
\hline D2 & 79 & $\mathrm{~F}$ & Osteoporosis & Denosumab & No & -3.7 & -3.1 \\
\hline D3 & 72 & $\mathrm{~F}$ & Osteoporosis & Denosumab & No & -3.2 & -2.8 \\
\hline D4 & 63 & $\mathrm{~F}$ & Osteoporosis & Denosumab & No & -3.7 & -2.9 \\
\hline D5 & 69 & $\mathrm{~F}$ & Osteoporosis & Denosumab & No & -2.5 & -2.0 \\
\hline D6 & 75 & $\mathrm{~F}$ & Osteoporosis & Denosumab & No & -4.5 & -1.9 \\
\hline D7 & 60 & $\mathrm{~F}$ & Osteoporosis & Denosumab & No & -1.5 & -2.8 \\
\hline D8 & 78 & $\mathrm{~F}$ & Osteoporosis & Denosumab & No & -2.6 & -2.3 \\
\hline D9 & 72 & $\mathrm{~F}$ & Osteoporosis & Denosumab & Yes & -2.6 & -2.2 \\
\hline D10 & 81 & $\mathrm{~F}$ & Osteoporosis & Denosumab & Yes & -1.5 & -2.9 \\
\hline P1 & 64 & $\mathrm{~F}$ & Osteoporosis & Teriparatide & Yes & - & -0.8 \\
\hline P2 & 79 & $\mathrm{~F}$ & Osteoporosis & Teriparatide & Yes & -0.9 & -2.2 \\
\hline P3 & 81 & $\mathrm{~F}$ & Osteoporosis & Teriparatide & Yes & - & - \\
\hline P4 & 79 & $\mathrm{~F}$ & Osteoporosis & Teriparatide & Yes & -4.3 & -2.7 \\
\hline P5 & 84 & $\mathrm{~F}$ & Osteoporosis & Teriparatide & Yes & -2.9 & - \\
\hline P6 & 91 & $\mathrm{~F}$ & Osteoporosis & Teriparatide & Yes & -3.3 & -3.8 \\
\hline P7 & 77 & $\mathrm{~F}$ & Osteoporosis & Teriparatide & Yes & -1.4 & -2.1 \\
\hline P8 & 71 & $\mathrm{~F}$ & Osteoporosis & Teriparatide & Yes & -4.5 & -2.8 \\
\hline
\end{tabular}

SF1c transcription start site (Supplementary Fig. S1). Overall, the correlation between the methylation levels of the two serum aliquots analyzed was $r=0.66$. The coefficient of variation was $17 \%$. All samples from the same individual were run in the same assay to minimize batch effects.

\section{Serum sclerostin and bone turnover markers}

Serum sclerostin levels were analyzed with an enzymelinked immunosorbent assay kit (Teco Medical Group). Sensitivity was $0.05-3 \mathrm{ng} / \mathrm{mL}$. Interassay coefficient of variation $(\mathrm{CV})$ was $16.8 \%$.

The levels of the bone turnover markers $\mathrm{N}$-terminal propeptide of type I procollagen (P1NP) and C-terminal telopeptide of type I collagen ( $\beta$-crosslaps) were measured with automatized chemiluminescence assays (IDS-iSYS MultiDiscipline Automated Analyzer) in serum samples taken after overnight fasting. Sensitivity was 1 and $0.033 \mathrm{ng} / \mathrm{mL}$, respectively. The intra-assay $\mathrm{CV}$ was $2.9 \%$ and $4.6 \%$ for $\mathrm{P} 1 \mathrm{NP}$ and $\mathrm{B}$-crosslaps assays, respectively. All samples from the same individual were run in the same assay to minimize batch effects.

\section{Statistical analyses}

Differences in the methylation levels of the different DNA sources from the same patient were analyzed by paired $t$-test. In addition, the correlation between these DNA methylation levels was analyzed by Pearson correlation tests. DNA methylation levels and bone turnover biomarkers before and after antiosteoporotic therapies were compared by paired $t$ test. $p$-Values $<0.05$ were considered statistically significant.

\section{Results}

SOST gene methylation in bone, blood cells, and serum

In a preliminary exploratory analysis, we did not find significant differences between patients with hip fractures and those with hip osteoarthritis. Therefore, the results of both groups were combined. The average methylation level of the SOST promoter was very similar in serum cfDNA and in bone-derived DNA $(79 \%-12 \%$ vs. $82 \%-7 \%, n=33)$. However, the average methylation level was much higher in blood cell DNA, 87\% - 10\% $(n=10)$. Pairwise comparisons revealed statistically significant differences between blood and serum cfDNA ( $p=0.0011)$ and between blood and bone DNA ( $p=0.0106)$, but not between serum cfDNA and bone DNA $(p=0.11)$. The analysis of individual CpGs confirmed higher methylation of CpG1 and CpG2 in blood cell DNA in comparison with either serum or bone DNA, whereas CpG3 was similarly methylated in DNA from the three sources (Fig. 1). Moreover, when all results were combined, there bF1 was a better correlation between the bone DNA and serum cfDNA methylation levels $\left(r=0.77 ; p=8.4 \cdot 10^{-7}\right)$ than between bone DNA and blood cell DNA methylation levels $\left(r=0.59 ; p=5.1 \cdot 10^{-4}\right)$. 

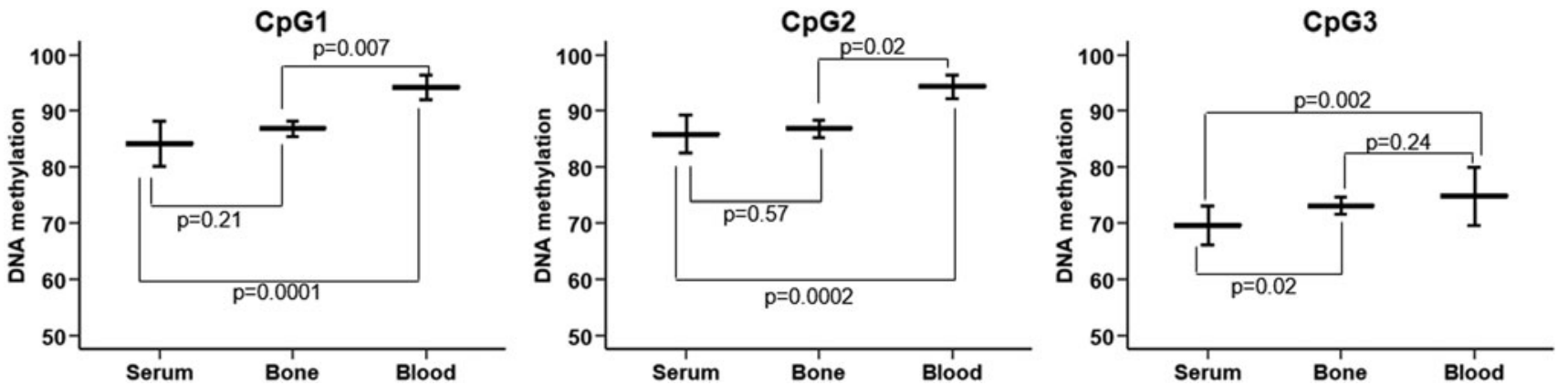

FIG. 1. The average and confidence interval of the DNA methylation levels from three CpG sites of the SOST promoter in serum cfDNA, bone-derived, and blood cell DNA. In each CpG site, differential analysis ( $t$-test) is shown between the three different DNA sources. cfDNA, cell-free DNA.
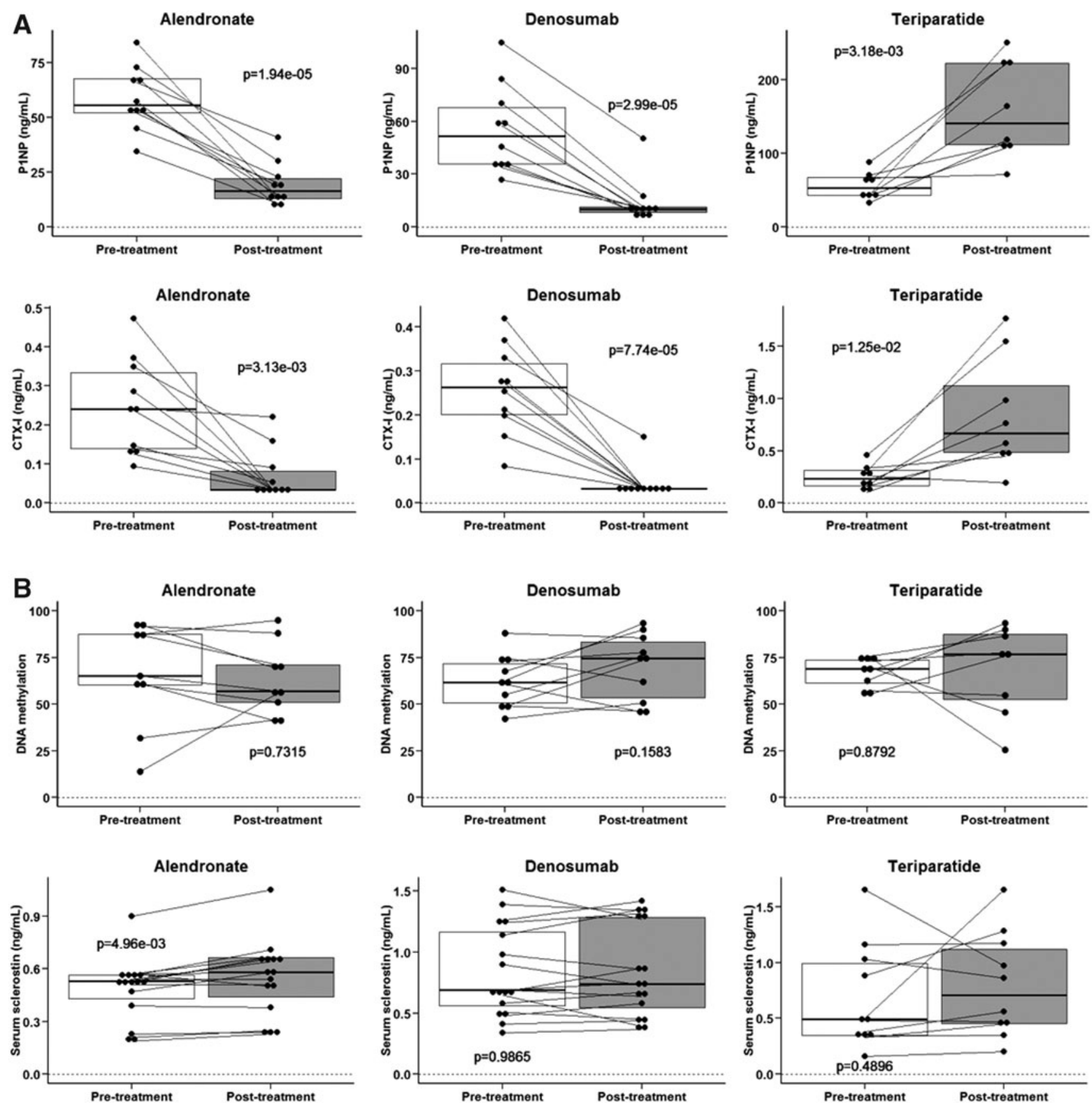

FIG. 2. (A) Serum levels of the bone turnover markers P1NP and b-crosslaps after 6 months of antiosteoporotic therapy with alendronate, denosumab, and teriparatide. (B) Serum levels of the SOST promoter methylation in cfDNA and serum sclerostin levels after 6 months of antiosteoporotic therapy. P1NP, propeptide of type I procollagen. 


\section{Serum biomarkers and response to therapy}

We found that serum P1NP and ß-crosslaps levels decreased as expected after antiresorptive therapy with alendronate ( $p=1.94 \cdot 10^{-5}$ and $p=3.13 \cdot 10^{-3}$, respectively) and denosumab $\left(p=2.99 \cdot 10^{-5}\right.$ and $\left.p=7.74 \cdot 10^{-5}\right)$. On the other hand, P1NP and crosslaps serum levels increased after 6 months ofteriparatide therapy $\left(p=3.18 \cdot 10^{-3}\right.$ and $p=1.25$ -

F2 c $10^{-2}$, respectively) (Fig. 2A).

Although, bone turnover markers changed dramatically as expected, we did not find significant changes in either SOST methylation in cfDNA or serum sclerostin levels after 6 months of antiosteoporotic therapy (Fig. 2B).

\section{Discussion}

SOST expression is not detected in peripheral blood cells (unpublished data). Consistent with that observation, in this study, we found that SOST promoter was highly methylated in DNA isolated from blood cells. However, SOST methylation in circulating cfDNA was significantly lower, thus suggesting that blood cells are not the major source of that cfDNA. In fact, we found similar levels of methylation at the SOST promoter in serum cfDNA and bone-derived DNA. Individually, there was a positive correlation between bone and cfDNA methylation levels. In general, the methylation level of bone DNA was better correlated with cfDNA methylation than with blood cell methylation. Overall, those results suggest that cfDNA in serum could mainly come from bone, at least in our samples, which included patients with skeletal abnormalities. Hence, our results point at cfDNA methylation as an index of bone cell activity, and specifically of bone sclerostin synthesis.

To further explore the association between cfDNA methylation and bone status, we analyzed cfDNA in patients treated with antiresorptive or anabolic drugs. Unfortunately, we did not observe significant changes in serum sclerostin in response to therapy, and, in line with that, we did not find changes in cfDNA methylation either. These results were not the consequence of lack of adherence or drug resistance, as we observed marked changes in bone turnover markers after therapy, as expected. Our results differ from other studies showing a decrease in serum sclerostin following teriparatide therapy (Drake et al., 2010). However, that is not a universal finding and other investigators have reported a lack of effect of treatment on serum sclerostin (Gossiel et al., 2018). Differences in the assays used, the length of therapy, or patients' characteristics may explain those conflicting results. We just found a statistically significant, but quantitatively very small increase in serum sclerostin in alendronate-treated patients, whose biological relevance is unclear.

Our study has some limitations. First, the relative small sample size limits the statistical power to find significant differences. This is particularly relevant for the analysis of changes in the methylation level after drug therapy or potential differences between patients with osteoarthritis and those with hip fractures. Also, we cannot exclude the possibility of methylation changes in regions of the SOST gene located outside the studied region. From the point of view of its clinical application, the analysis of cfDNA methylation is technically laborious and with higher coefficient of variation than the currently used bone turnover markers. Thus, it is unlikely to become a substitute for them, but it could add some useful information about the regulation of some genes at the bone tissue. Most cfDNA may be associated with microvesicles formed during cell degeneration. However, studies with cancer patients suggest that cfDNA is not a simple marker of tumor burden or cell death and may indeed be determined by tumor metabolism by mechanisms not elucidated yet (Kustanovich et al., 2019; Malkin and Bratman, 2020). Thus, it is tempting to speculate that the methylation pattern of bone-related genes could also reflect, at least in part, bone metabolism. However, further larger studies are needed before to support its potential role in research studies and clinical practice.

There are conflicting reports about the role of sclerostin in the cardiovascular system, with some, but not all studies showing an association between serum sclerostin and cardiovascular risk (Mathold et al., 2018; Novo-Rodr'iguez et al., 2018). Several investigators have reported sclerostin expression in atherosclerotic, calcified lesions of the arterial wall. This seems particularly clear for patients with advanced kidney disease (Nitta et al., 2018). Thus, although we did not include patients with renal failure, we cannot completely exclude the possibility that unmethylated SOST DNA comes, in part, from the vascular wall.

In conclusion, in this study, we showed that SOST methylation in serum cfDNA does not reflect the methylation level in DNA from blood cells, which is consistent with an extrahematological origin of this cfDNA. Our results indicate that methylation status of SOST cfDNA could reflect SOST methylation levels in bone. To our knowledge, this is the first study addressing the possibility of using serum cfDNA as a "liquid biopsy" in non-neoplastic bone disorders. Further studies showing a correlation between bone SOST changes and SOST methylation in cfDNA are needed to confirm its potential role as a bone biomarker.

\section{Conclusions}

DNA methylation levels of the SOST promoter in serum cfDNA are lower than in blood cell DNA and similar to bone DNA, suggesting that serum cfDNA originates, in part, in bone cells. However, we did not find significant changes in the levels of serum sclerostin or DNA methylation levels after therapy with bone-active drugs. Interestingly, serum cfDNA of the SOST promoter does not reflect the methylation level in peripheral blood cells. Thus, it is worth studying the role of cfDNA as a bone biomarker.

\section{Author Disclosure Statement}

No competing financial interests exist.

\section{Funding Information}

This study was supported by two grants from Instituto de Salud Carlos III (PI12/615 and PI16/915), through a program potentially co-funded by FEDER Funds from the European Union.

\section{Supplementary Material}

Supplementary Figure S1 


\section{References}

Biró O, Rigó J Jr., Nagy B (2018) Noninvasive prenatal testing for congenital heart disease-cell-free nucleic acid and protein biomarkers in maternal blood. J Matern Fetal Neonatal Med 5:1-11.

Connolly ID, Li Y, Gephart MH, Nagpal S (2016) The "Liquid Biopsy": the role of circulating DNA and RNA in central nervous system tumors. Curr Neurol Neurosci Rep 16:25.

Delgado-Calle J, Sañudo C, Bolado A, et al. (2012) DNA methylation contributes to the regulation of sclerostin expression in human osteocytes. J Bone Miner Res 27:926-937. Delgado-Calle J, Sato AY, Bellido T (2017) Role and mechanism of action of sclerostin in bone. Bone 96:29-37.

Drake MT, Srinivasan B, Mödder UI, et al. (2010) Effects of parathyroid hormone treatment on circulating sclerostin levels in postmenopausal women. J Clin Endocrinol Metab 95: 5056-5062.

Goldwaser T, Klugman S (2018) Cell-free DNA for the detection of fetal aneuploidy. Fertil Steril 109:195-200.

Gossiel F, Scott JR, Paggiosi MA, et al. (2018) Effect of teriparatide treatment on circulating periostin and its relationship to regulators of bone formation and BMD in postmenopausal women with osteoporosis. J Clin Endocrinol Metab 103:1302-1309.

Kustanovich A, Schwartz R, Peretz T, Grinshpun A (2019) Life and death of circulating cell-free DNA. Cancer Biol Ther 20: $1057-1067$.

Lehmann-Werman R, Magenheim J, Moss J, et al. (2018) Monitoring liver damage using hepatocyte-specific methylation markers in cell-free circulating DNA. JCI Insight 3: e120687.

Malkin EZ, Bratman SV (2020) Bioactive DNA from extracellular vesicles and particles. Cell Death Dis 11:584.
Mathold K, Wanby P, Brudin L, et al. (2018) Alterations in bone turnover markers in patients with noncardio-embolic ischemic stroke. PLoS One 13:e0207348.

Nitta K, Hanafusa N, Okazaki M, et al. (2018) Association between risk factors including bone-derived biomarkers and aortic arch calcification in maintenance hemodialysis patients. Kidney Blood Press Res 43:1554-1562.

Novo-Rodr' 1guez C, Garc' 1a-Fontana B, Luna-Del Castillo JD, et al. (2018) Circulating levels of sclerostin are associated with cardiovascular mortality. PLoS One 13:e0199504.

Reppe S, Noer A, Grimholt RM, et al. (2015) Methylation of bone SOST, its mRNA, and serum sclerostin levels correlate strongly with fracture risk in postmenopausal women. J Bone Miner Res 30:249-256.

Weivoda MM, Youssef SJ, Oursler MJ (2017) Sclerostin expression and functions beyond the osteocyte. Bone 96:45-50. Yamashita K, Hosoda K, Nishizawa N, et al. (2018) Epigenetic biomarkers of promoter DNA methylation in the new era of cancer treatment. Cancer Sci 109:3695-3706.

Zou J, Duffy B, Slade M, et al. (2017) Rapid detection of donor cell free DNA in lung transplant recipients with rejections using donor-recipient HLA mismatch. Hum Immunol 78: 342-349.

Address correspondence to: Jose' A. Riancho, MD Department of Internal Medicine Hospital Universitario Marqués de Valdecilla-IDIVAL University of Cantabria Santander 39011 b AU5 Spain

E-mail: jose.riancho@unican.es 


\section{AUTHOR QUERY FOR GTMB-2020-0172-VER9-REAL 1P}

AU1: Please note that gene symbols in any article should be formatted as per the gene nomenclature. Thus, please make sure that gene symbols, if any in this article, are italicized.

AU2: Please identify (highlight or circle) all authors' surnames for accurate indexing citations.

AU3: Please expand the first initials in the author names "M. Isabel Perez-Nuñez, M. Teresa Garcia-Unzueta, and M. Carmen Valero.",

AU4: The Clinical Trial Registration number, if applicable, should be included at the end of the abstract.

AU5: Please mention the postal address in corresponding author's address. 